\title{
Swarm-based Active Tunable Routing for Overhead Reduction in Multiservice Networks
}

\author{
Constandinos X. Mavromoustakis and Helen D. Karatza \\ Department of Informatics \\ Aristotle University of Thessaloniki \\ 54124 Thessaloniki, Greece \\ \{cmavrom,karatza\}@csd.auth.gr
}

\begin{abstract}
The explosive growth of multimedia and other bandwidth intensive applications has resulted a rapid increase in the size of the traffic loads that needs to be supported by modern networks. This paper describes the combination of the active network concept with swarm based control method for optimized multiclass QoS priority routing. This hybrid scenario uses the bandwidth clustering approach (triggered clusters). The packets follow a predetermined path initialized and allocated by swarm based packets, blindly and without any global knowledge of network conditions. In this work this mechanism is associated with the triggered pheromone explored at different simulation sessions and does not require message brokers to fulfill multiclass QoS demands. The pheromone enforcement model that is indicated increases the overall response of the system. Simulation results depict the performance and robustness of the scheme in terms of the end-to-end delay, available bandwidth and packet loss under different traffic measures.
\end{abstract}

\section{Introduction}

Maintaining efficient routing and simultaneously enable resource allocation onto communication channels is not an easy task. Particularly if applied in today's hybrid and complex networks. The concept of active networks provides the capability of on demand analysis and modification of packets messages flowing through the internal nodes of the network. By combining various hybrid schemes of different active agent-based methods the overall performance of the system is enhanced. The objective of the generic concept of active agent-based techniques is to achieve high resource utilization and to reduce user contention for network resources. The mechanism used for load balancing and resource allocation has to avoid overloaded nodes such that transmission delays (latencies) are minimized, whereas alternative routes should be activated whenever load conditions are drastically changing.

While there are often models and efficient algorithms facing allocation problems in deterministic systems (typically formulated as matching or flow optimization problems) solutions for the networks with stochastic components are not straightforward. Decentralized schemes [3, 6] allow the central control to be applied locally to a part of a network enabling all "local" supervisors to cooperate deploying a decentralized exchange of information. Indeed many distributed systems are unreliable and subject to random failures of their components. Examples of such systems are power grids where the distribution ability of the network can be affected by demand overloads and other random events or various transportation/information networks subjected to congestions and intermittent failures. Thus optimally designed systems have to offer end user reliability and integrity.

This work examines a hybrid combination of active network technique. The scenario in this work uses embedded swarm based packets [8] that are delay sensitive and marks these packets as prioritized. As it will be explained this hybrid method provides a model for active and distributed network data flow organization and also continuous resource reservation on demand. As a part of this method the bandwidth (BW) clustering mechanism is activated for assigning a certain BW onto communication channels. Furthermore the BW clustering scenario is associated with a pheromone explored session mechanism for reinforcement of additive pheromone onto available channels. A number of metrics are considered that are associated with network performance. Additional 
metrics for handling multimedia streaming (MMs) are presented, dealing with the reliability and QoS offered by the proposed scheme.

The organization of the paper is as follows: In section 2 a description of the basic principles of the swarm-based scheme and adaptive resource allocation is presented with extensive use of active multiclass bandwidth clustering method. Section 3 draws the simulation results and conclusions and suggestions for future research are summarized in section 4 .

\section{Adaptive swarms for resource allocation and efficient flow control}

Studies $[4,6]$ have shown that an ant-like mobile agent algorithm could be applied to a network with significant optimization of the QoS metrics of the network. In [3, 4], Dorigo and Gambardella used the metaphor of trail laying by ants to certain combinatorial optimization problems. Several agentoriented approaches $[4,6,12]$ have recently been proposed that appeal to principles extracted from Swarm Intelligence (SI).

Swarm network routing is based on insect colonies which exhibit a simple behavior for their communication and living. They are represented in the network as artificial agents that bias the network collecting useful information for the whole environment through their hormones called pheromones. In previous researches $[8,12]$ a hybrid (proactive and reactive) agent behavior is developed in which ants are adapting their communicational behavior to network circumstances, simultaneously splitting themselves for passing information to neighbor nodes.

One of the major challenges of the service/resource allocation problem is to find algorithms that are reactive and deliver reliable high quality solutions. Sources usually react by reducing their flow. Indeed, this is a response to congestion, but it has the unavoidable side effect of reducing the throughput. Figure 1 shows the relation between traffic-incoming load, flow control and final throughput. Nodes S and $\mathrm{D}$, are linked via different service platforms (MSPMultiService Platforms) that affect the underlying discrete flow control-for any given time t- which results fluctuation in throughput response of the system.

The proposed adaptive method allows nodes to change at any time their state depending entirely on packets channel characterization. This scenario is examined under certain conditions as discussed in the following section.

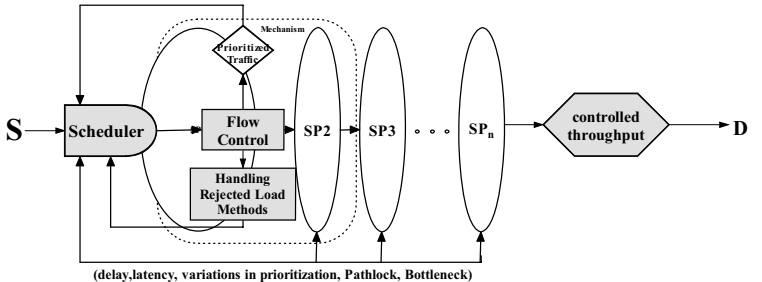

Figure 1: Relation between traffic-incoming load, flow control and final throughput. $S$ and $D$ are linked via different service platforms (MSP-MultiService Platforms).

\subsection{Embedded swarm based active packets for resource allocation}

2.1.1 Active network approach and resource discovery

In an active network, packets are more than just passive data. They are programs executed by the nodes that are passing the packets along or embedded in packets that influence control decisions. This facility allows packets to make on-the-fly decisions about how to route themselves. Tennenhouse et al [2] provides a survey of this work. Active network's approach is motivated by both lead user applications, which perform user-driven computation at nodes within the network.

Current resource discovery approaches for offering optimized QoS have been designed primarily for small networks or for networks where dynamic updates are not common or frequent. Hence, they do not scale well when the number of resources grows, and updates are common. Some of the most popular service discovery systems are Jini from Sun Microsystems, SLP [5], INS (Intentional Naming System) [10], and SDS [9]. In those systems, services advertise their descriptions to resolvers-sometimes to intermediate nodes- which act as resource directories. In turn resolvers allocate the dedicated streaming packets to a link where available resources are adequate to efficiently forward streams from a node to another.

In this work resource capacities are provided locally to where demands occur, avoiding cross-network traffic. This active technique is proved to be very adaptive and can rapidly respond to changes in the environment while significantly reduces the generated network overhead.

\subsubsection{Swarm based active network}

Active packets and nodes can perform different computations reactively. The encapsulation abilities of such a network enable advanced mechanisms for end to end communication to scarce or sensitive resources. Traditional packet headers are replaced with control programs in order to make on the fly decisions. 
The swarm based system approach associates pheromone trails to features of the solutions of a combinatorial problem, which can be seen as a kind of adaptive memory of the previous solutions. In our implementation each packet-agent launches in the network and influences the pheromone table by increasing or reducing the entry for the proper destination using antipheromone [8]. The pheromone quantity represents the available end-to-end resources. The pheromone table at each node $N_{i}$ with $k(i)$ neighbors can be measured as:

$R_{i}=\left[r_{1, m}^{i}\right]_{n-1, k(i)}$ with $n-1$ destinations and $k$ next nodes for $N_{i}$

In the network ants are walking according to probabilities assigned in pheromone tables and they are visiting one node at every time step. In this way ants increase the entry in the pheromone table corresponding to the node from which they came from:

$$
\mathrm{P}=\frac{P_{\text {old }}+\Delta P}{1+\Delta P}
$$

where $\Delta P$ is the quantity of pheromone increased and $P_{\text {old }}$ is the previous entry. The other entries in the table of this node are decreased accordingly following the formula:

$$
P=\frac{P_{\text {old }}}{1+\Delta P}
$$

Routing tables contain a two-way pheromone table parameters (bi-directional links with different capacity) which are maintained in each node, and are expressed as:

$$
P_{k(i)}^{i_{t} \mapsto n_{t}} \text { and } P_{k(i)}^{n_{t} \mapsto i_{t}}
$$

where $k(i)$ are the next nodes for $N_{i}, n$ is one of the $n-1$ possible destinations and $n_{t}$ is the possible next node at a certain time step.

Metaheuristic techniques allow the formulation of an efficient strategy for providing a complete solution for Multiservice network. These techniques effectively reduce the total cost in terms of generated overhead. All probabilities are thresholded [8] between $\frac{1}{(\text { number_of_neighbors })^{2}}$ and 0.75 in order to prevent the pheromone saturation state.

The route, where intermediate nodes have large pheromone quantities, is selected as the best-chosen path. If the destination can be reached on a hop-by-hop look-up table method, the route is valid otherwise the

\footnotetext{
Nodes can be both endpoints (can be source(s) and destination(s)) and switches (can perform routing functions).
}

packet is blocked [8] and lost. Routing table entries updates are measured according to the following:

$$
r_{i-1, s}^{i}(t+1)=\frac{r_{i-1, s}^{i}(t)+\delta r}{1+\delta r}
$$

where $\delta r$ is the step size parameter and $s$ is the source node. Similarly for all neighbors to $i, r_{n}^{i}(t)$ is found that:

$$
r_{n, s}^{i}(t+1)=\frac{r_{n, s}^{i}(t)}{1+\delta r}, n \neq i-1
$$

As mentioned ants deposit pheromone trails on the path. In turn the ants that follow collect this quantity by measuring the density. In our model we correlated the pheromone quantity with the different sessions of exploration of pheromones. In an initial session $i$, an explorer agent $m$ chooses probabilistically session $j$ as the next quantity by using the following:

$$
P_{m}(i, j)= \begin{cases}\frac{\left[\tau_{(i, j)}\right]^{\alpha} \cdot\left[\eta_{(i, j)}\right]^{\beta}}{\sum_{k \in S_{m}(i)}\left[\tau_{(i, j)}\right]^{\alpha} \cdot\left[\eta_{(i, j)}\right]^{\beta}} & \text { if } j \in S_{m}(i)(6.1) \\ 0 & \text { otherwise }\end{cases}
$$

where

$\tau_{(i, j)}$ : Intensity measure of the pheromone deposited by each ant on the path $(i, j)$. The intensity changes during the run of the program.

$\alpha$ : Intensity control parameter. It is entirely based on the connectivity maintenance between adjacent nodes and the probability of the explorer ant to become unavailable in the process.

$\eta_{(i, j)}$ : Quality of the path $(i, j)$. This visibility, which remains constant during the run of the program, is determined by $\eta_{(i, j)}=1 / l_{(i j)}$, where $l_{(i j)}$ is the cost of move from session $i$ to the session $j$.

$\beta$ : Ability control parameter based on each ants collecting ability (resources availability).

$S_{m}(i)$ : The set of sessions that remain to be observed by ant $m$ positioned at session $i$.

Equation 6.1, which is conceptually inspired from [7], shows that the quality of the path $(i, j)$ is proportional to its shortness and to the highest amount of pheromone deposited on it (i.e., the selection probability is proportional to path quality).

Each packet has a state with a prioritization. According to this fact each packet can affect the quantity of deposited pheromone with the so called positive or negative enforcement as:

$$
P_{(i, j)} \stackrel{\text { active }}{\longleftarrow} P_{i+\delta(\operatorname{Pr})}+P_{i+\delta(\operatorname{Pr}(\tau))}
$$

where $\delta(\operatorname{Pr})$ is the reduced or increased pheromone values in the path according to priority degree of each packet, $\tau$ is the maximum time distance that is required to reach destination and $P_{(i, j)}$ is pheromone 
enforcement degree from a point $i$ to a point $j$ in the network.

In this way swarm packets and virtual ants have an interaction in the means that ants affect the routing tables while data packets influence the service rate of the traffic on nodes, which affects the ants with the delay mechanism. Equation 6.2 is trying either to provide a greater amount of pheromone or to reduce and balance the amount of pheromone in terms correlated to the prioritization degree of each packet that traverses the path. This swarm/agent-oriented approach encompasses the generic agent based concept which enables agents to move around the network, gathering information about the topology of the system and the traffic at a discrete time.

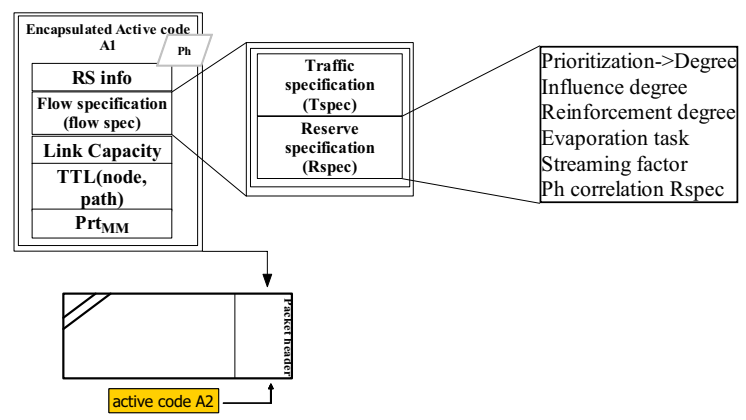

\section{Packet structure with Ph correlation with Rspec \\ Figure 2: Active swarm based packet's structure with the Pheromone correlation on Rspec field.}

Active swarm based packets are used in a network for internal communication and auto-configuration between packets and nodes. Roughly speaking active packets are self-contained piece of software that has the properties of autonomy and interaction. Packets make on the fly decisions about how to route themselves and learn (gather information) from their passing-by environment. Figure 2 shows the structure of swarm based packet's structure with the pheromone correlation on Rspec field.

A packet consists of a "flow spec" field and a "filter spec" field as in the well known Resource reSerVation Protocol (RSVP) [11]. Packets 'carry' traffic spec and path information from a source to destination and reserve information during their journey to destination. The RS info field of the encapsulated active code is the main swarm based active field in the packet's header. $\mathrm{RS}$ info field, in cooperation with other fields in both node and packet, can expose the determined quantity of pheromone for the selected source and destination. This pheromone quantity is based on the available endto-end resources [8, 12]. The flow spec specifies the desired QoS for the packet. The filter spec defines the set of data packets to receive the QoS defined by the flow spec. The entire service class in packet header (both A1 and active code A2 of fig.2) defines the desired QoS and beyond others, it describes the type of the data flow (priority degree of MM or don't care packets). In figure 2 the Rspec field is related to pheromone evaporation degree which is proportional with the streaming factor of each multimedia packet. It is essential to state out that the communication between nodes and packets is opaque to other network layers and that the prioritization degree is segmented into five levels [8].

\subsubsection{Resource reservation mechanism using bandwidth clustering method}

The bandwidth clustering approach is based on the available bandwidth on each data link in the network. Due to the unpredictable incoming traffic measurements that occur at any time in the network, the capacity of each channel (bandwidth) is reduced progressively with an increased flow of packets. Bandwidth clustering method is based on the idea of clustering nodes using different levels of bandwidth [8]. The clustering idea is based on whether the remaining capacity could efficiently be reserved by different streams to better utilize the path. Overloaded paths are also clustered but the remaining capacity will mark the cluster as a lower bandwidth cluster [8]. The bandwidth clustering method is associated with a cooperative learning and active environment, producing a robust and decentralized way for adapting link's changing capacities quickly.

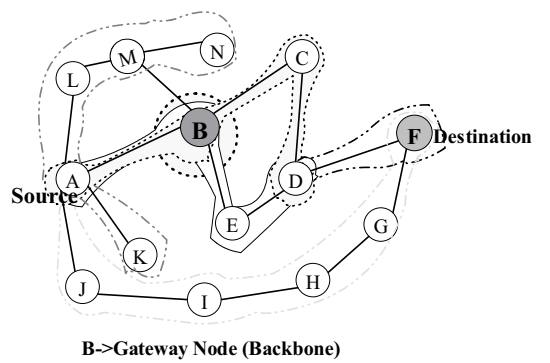

Figure 3: A random topology and the regions arising when clustering with respect to several levels of bandwidth.

Figure 3 illustrates the typical decision selections from a proper source node $\mathrm{A}$ to destination $\mathrm{F}$. There are many path options using different intermediate nodes and different clusters leading to F. A cluster is being constructed at each path having the available bandwidth of the channel with the lowest free remaining capacity (1).

$$
\operatorname{Max}\left(C_{A \mapsto D}\right)=B W
$$


In equation $7, \mathrm{BW}$ is the available bandwidth, which is determined by the lowest free remaining capacity in the path leading from source node to destination.

All nodes are informed and the clusters are constructed according to capacity reservation information spread by active swarms onto nodes. Swarm based routing decisions affect the bandwidth clustering method, which in turn affects bandwidth reservation.

To define the accurate resource allocation in terms of bandwidth the updated entries of the pheromone table of node $n$ should satisfy the following:

$$
p_{b}=\sum_{i} r_{n, s}^{i}=100 \%=\text { full } \_B W
$$

The BW clustering scheme will enable the reduction of lookup tables and generated overhead, thereupon additional queries to neighbors are avoided.

\subsubsection{Constructed clusters' recreation frequency and triggering}

After consecutive network flows (steady state) it is shown [12] that network is biased overcoming the transient state. Thus paths are marked and ready for the creation of each cluster to host a bounded capacity. Beyond the path marking shown in [12] we used cellular automata (CA) to overcome the pathlock [8]. For instance node $N_{A}$, uses CA simply to exchange information with the neighboring nodes examining ${ }^{2}$ whether its neighbors have at least a single link leading to the proper destination (that has higher remaining capacity). If these links of $N_{A}$ 's neighbor are not empty $N_{A}$ remains 'alive' and the cluster is not destroyed. Otherwise the cluster is obliterated.

Triggering with real traffic (swarm based active packets) is an action in order to set different paths in combination with the co-operative agents and CA comparison in each node. Each time a packet is transmitted from a source to a destination, information regarding the cluster of which the packet itself is a part of, is exchanged between the packet and nodes. This mechanism occurs in a dynamic way (pheromones extraction) exchanging information about the available bandwidth at each time step. Each cluster has a threshold $S_{\text {cap }}$, and provides an active environment with a proper QoS. Some thresholds for bandwidth should be chosen so that nodes could be dynamically clustered in areas called "blocking areas". Every node in the path is grouped and belongs to the blocking area

\footnotetext{
Examination is performed within routing tables.
}

labeled with $X \mathrm{Mb} / \mathrm{s}$, if at least one route with as much free bandwidth between nodes exists. Thus if the threshold $S_{\text {cap }}$, where $S_{\text {cap }}<$ link_available_ $B W$, is overtaken, meaning that the cluster has reached or passed the overloaded value, then once again the cluster is obliterated and recreation procedure takes place.

\subsection{Adaptive resource allocation with reliable traffic flows in constructed clusters}

Each node measures the number of links at the beginning of each time step $\tau$ and the number of its broken links $\tau_{\text {Tot }}$ (if any). The rate of broken links is equal to: $\frac{\tau_{b}}{\tau_{T o t}}$, where $\tau_{b}$ is the number of node's broken links. One way to evaluate reliability is by using the following notation:

$$
\left(1-\frac{\tau_{b}}{\tau_{T o t}}\right)^{\mathrm{T}_{i} \cdot N}
$$

where $\mathrm{T}$ is the time steps that have passed since the creation of the cluster(i), and $\mathrm{N}$ is the number of nodes in the cluster.

According to equation 9 we have measured the total path reliability notation and the link survivability factor as follows:

$$
R=\left(1-\frac{\tau_{b}}{\tau_{T o t}}\right)^{\mathrm{T}_{i} \cdot N+\sum_{k=1}^{h o p s} \frac{k-1}{R f}} \text {, number of hops }>1
$$

where $R f$ is the link capacity refreshment factor and $i$ is the number of links in the path. In this way we evaluate the total reliability by using equation 10 above.

\subsection{Network generated overhead and Quality of Service (QoS)}

The swarm-based scenario does not use specific control packets since ants pursue the control by being a part of the packets (smart-active packets). Therefore embedded swarm agents map and control the traffic at any time during transmission in the network, and consequently overhead is potentially reduced. The inexistence of generated overhead is a result of the non-transmitted routing table values or other information blocks to neighbors or to all nodes of the network. In essence it increases the efficiency without the need for packet tunneling. Additionally no message brokers are needed to fulfill QoS demands issued by applications. 


\section{Simulation results and discussion}

To evaluate the described scenario, exhaustive simulations were performed to a partially meshed 100 node network. Nodes capacity has been chosen to be relatively high on each node's buffer $(680 \mathrm{~kb}<1 \mathrm{MB})$. Network's performance is examined through a number of various metrics that characterize the efficiency of the proposed scheme. In the implementation of the bandwidth clustering scheme different traffic input streams have been tested ${ }^{3}$. The evaluation took place for different levels of prioritization (marked 15(highest priority)) and 'don't care' packets. The network traffic is modeled by generating constant bit rate (CBR) flows. Each source node transmits one 512bytes ( $\sim$ Kbits-light traffic) packet. In the described scenario link capacity is $6 \mathrm{Mbps}$ (bi-directional) each. Packet's requests are routed only once using the saved values on nodes. Paths are chosen according to pheromone values allocated by ants, and are set to be Hamiltonian (only once allocated to prevent packet's revisiting and link's saturation).

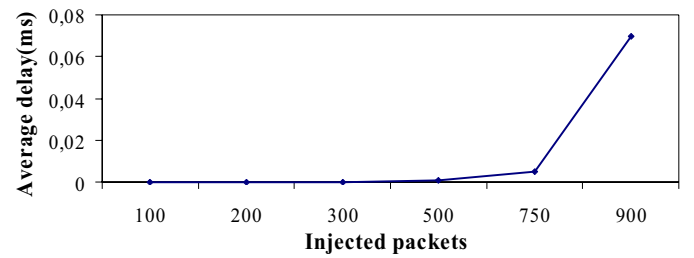

Figure 4: Average packet delay versus the number of injected packets.

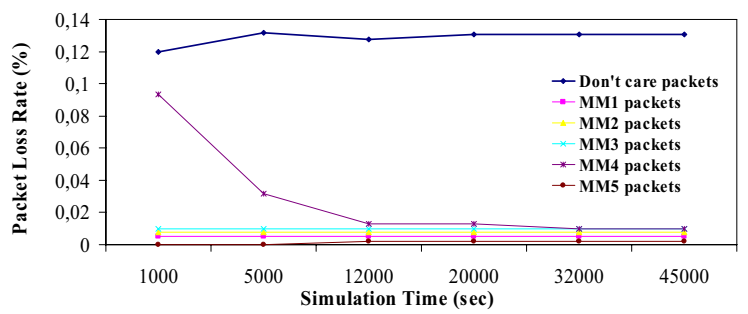

Figure 5: Packet Loss rate for different prioritization degrees of MM packets using the reinforcement concept.

In figure 4 the average packet delay with the number of injected packets in the network is illustrated. As the number of packets increases the average packet delay increases slightly. After consecutive simulations for this metric it has been shown that when the number of injected packets reaches $\approx 600$, the average packet delay increases dramatically. This occurs due to capacity limitations

\footnotetext{
Streams included Light Traffic(CBR randomly selected $\sim 4 \mathrm{Kbits})$ and Heavy Traffic (4• Light Traffic)
}

that bind each node. Swarm based active scheme shows that in order to reject the incoming packet, data flow scheduler and flow specification fields are responsible to cache locally the packet to node. This results in significant delay but negligible compared with the case of packet loss shown in figure 5 .

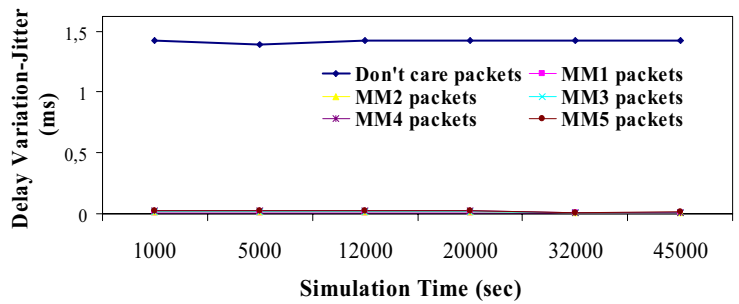

Figure 6: Delay variations for different grade of prioritization of packets, using the reinforcement concept.

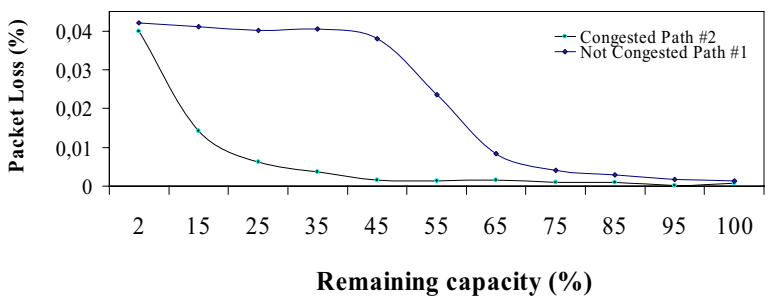

Figure 7: Percentage of packet loss with the mean remaining capacity in the communication channels.

Figure 5 illustrates packet loss rate for different grade of prioritization of MM packets as well as for "don't care" packets. "Don't care" packets have the highest packet loss rate because no prioritization takes place using the swarm based active scheme. Prioritized packets in real time could be video streams where packet loss and delay could be disastrous for the offered QoS to end users. From figure 5 we can discriminate that the packet loss rate for any of the MM 1-5 prioritized packets is relatively low compared with that of "don't care" packets. Additionally the prioritized packets MM-4 and MM-5 are behaving almost the same for packets loss rate.

In figure 6 the delay variations (or jitter) for different grade of prioritization of MM packets are shown. Once again the swarm based active scheme enables active prioritization of MM packets which cause significant reduction in the jitter parameter. Jitter is almost the same for low prioritization MM packets (MM-1) and for high prioritization (MM-4, MM-5). Active swarm based scheme proves to be robust in delays where sensitive prioritized packets are scheduled to be transmitted in bounded end-to-end delay. 


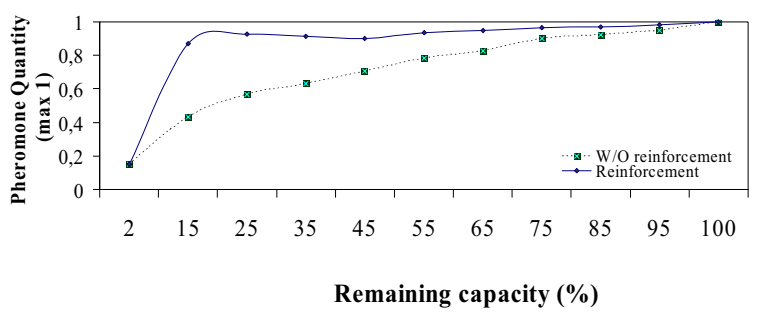

Figure 8: Impact of pheromone quantity with the manipulation of the mean remaining capacity in the communication channels.

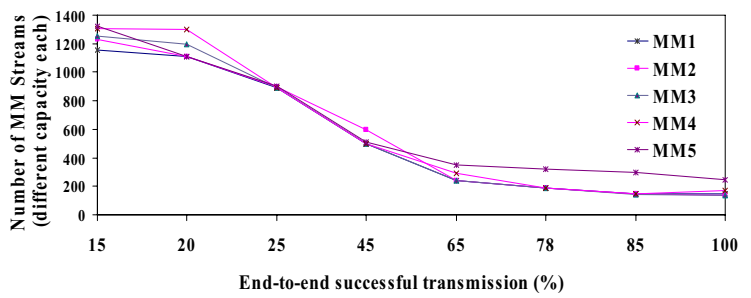

Figure 9: Service tunability for different priority degrees with and without pheromone reinforcements.

In figure 7 the percentage of packet loss with the mean remaining capacity is shown. The bottleneck concept -as in [8]- is adopted for this measure in order to evaluate the inner interconnection capacities between nodes. Two different measures were taken: for congested links and for non congested links. Noncongested links, which have less than $<30 \%$ remaining capacity in each node's buffer, seem to behave as expected. The metric for congested links shows the robustness characteristics and the activeness of the swarm based scheme. For almost $80 \%$ of each node's utilized buffer this scheme presents significantly lower values for packet loss rate.

Figure 8 presents the impact of pheromone quantity with the manipulation of the remaining capacity in the communication channels. Two experiments were used for this metric: without reinforcement and with reinforcement. It is evident that with the reinforcement scheme, the utilization of channels is more efficient. This occurs because of the extra release of capacity with the pheromone (path) reinforcement.

Figure 9 shows the service tunability for different priority degrees of MM packets. Each prioritized MM packet has different degree of prioritization to reach a destination. However MM-5 packets behave almost the same as MM-1 packets. This means that the swarm based active scheme enables localized prioritizations of packets to nodes. Thus when packets are marked as prioritized this scheme "distributes" this prioritization along the paths and influences other MM packets to follow the same path.
Figure 10 presents the percentage of successful reception with the number of different created paths. The successful reception ratio remains at high levels when the number of created clusters is relatively small, compared with the total number of created clusters. When the number of different created paths reaches the $70 \%$ of the total number of created clusters (about 200 newly created clusters), then the successful reception percentage drops proportionally with the increased number of created clusters. Although path lock with this measure is optimized, the successful reception percentage shrinks. This is due to the bounded end-toend limitations for the correct packet delivery where packets "follow" different paths with higher switching delay.

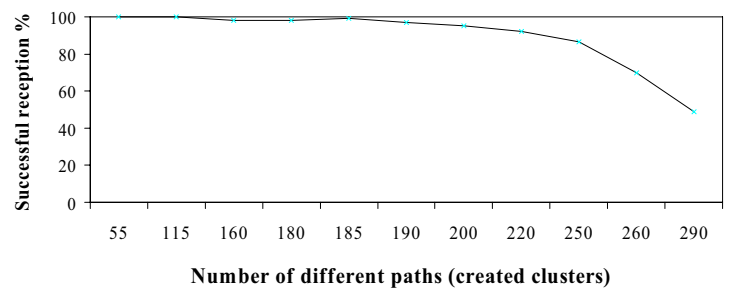

Figure 10: Percentage of successful reception with the number of different created paths.

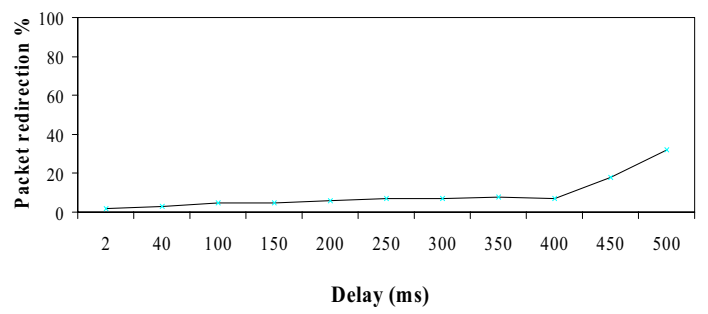

Figure 11: Percentage of redirected packets with the corresponding delay (ms).

Figure 11 shows the percentage of redirected packets for different paths than the initials allocated, with the corresponding delay (ms). It is evident that if the redirection mechanism takes place, then the time delay for the reliable end to end packet transmission increases. In figure 12 the percentage of the redirected packets is shown with the RReq (Route Request) parameter which is as a fraction of the total number of control packets generated. RReq is basic parameter for an accurate routing mechanism to take place. RReq is sent, when one node needs to send a message to another node that is not its neighbor (simply broadcasts a RReq message). RReq is a main element for controlling packets. From figure 12 it can be considered that if RReq is sent with high percentage (more than $50 \%$ of control packets), the path/packet redirection is about $40 \%$. This fact proves that swarm based active routing (which bounds the number of 
RReq packets sent), offers a robust and scalable way for on demand link resource allocation avoiding extra/additional control packet requests.

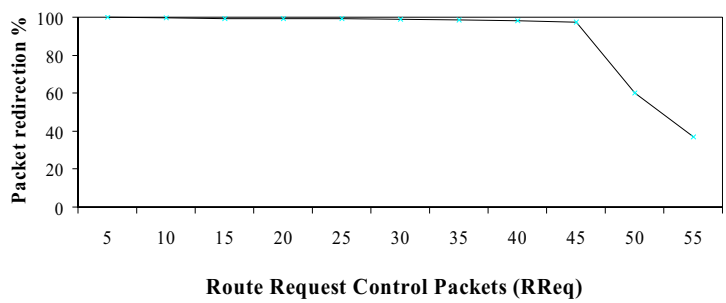

Figure 12: Percentage of redirected packets with the RReq parameter as a fraction of the total number of control packets generated.

\section{Conclusions and further research}

The primary objective of this paper is to explore the novel method of embedded swarms in an active scheme for network self-configuration. This scheme is suited for optimized QoS capacity allocations onto dynamically changing link's load. It bounds also a significant overhead reduction. The reliability factor in combination with the reinforcement factor onto communication links, enable accurate capacity allocation and bandwidth reservation on demand for any requested traffic. This method can better utilize saturated paths (pathlock) which is a common problem for heuristic methods.

This work can be extended for scalable interclass resource distribution to achieve higher network throughput. Additionally the pheromone concept can be alternatively modeled to represent other crucial cross referenced-issues of the system.

\section{References}

1 A. Chavez, A. Moukas, P. Maes, Challenger: A Multiagent System for Distributed Resource Allocation. Proceedings of the First International Conference on Autonomous Agents, Marina del Rey, California, United States, 1997, pp.323-331.

2 D. Tennenhouse, J. Smith, W. Sincoskie, D. Wetherall, and G. Minden, A Survey of Active Network Research. IEEE Communications Magazine, 1997, vol. 35, No 1, 80-86.

3 S. Appleby, S. Stewart, Mobile software agents for control in telecommunications networks, BT Technology Journal, 1994, vol. 12, pp. 104-113.

4 M. Dorigo, V. Maniezzo, A. Colorni, The Ant System: Optimization by a Colony of Cooperating Agents. IEEE Transactions on Systems, Man, and Cybernetics-Part B, 1996, vol. 26, No. 1, pp. 29-41.
5 E. Guttman, C. Perkins, Service Location Protocol, Version2. RFC2608. http: //www.ietf.org/rfc/rfc2608.txt (1999).

6 R. Schoonderwoerd, O. Holland, J. Bruten, L. Rothkrantz, Ant-based Load Balancing in Telecommunications Networks, Adaptive Behavior, vol. 5, 1997, pp. 169-207.

7 S. Fidanova, ACO Algorithm with Additional Reinforcement, In M. Dorigo, G. Di Caro and M. Sampels, editors, Ant Algorithms, Springer-Verlag, Berlin, Germany, 2002, vol. 2463, pp. 292-293.

8 C. Mavromoustakis, H. Karatza, Performance Evaluation of an On Demand Tunable Resource Allocation Apparatus Using Constrained Active Network. Journal of Simulation Modeling Practice and Theory, Elsevier, accepted for publication (to appear).

9 S. Czerwinski, B. Zhao, T. Hodes, A. Joseph, R. Katz, An Architecture for a Secure Service Discovery Service. Proceedings of the 5th annual ACM/IEEE international conference on Mobile computing and networking, Seattle, Washington, United States, 1999, pp. 24-35.

10 W. Adjie-Winoto, E. Schwartz, H. Balakrishnan, J. Lilley, The design and implementation of an Intentional Naming System(INS). Proceedings of the seventeenth ACM symposium on Operating systems principles, Charleston, South Carolina, United States, 1999, pp. 186-201.

11 L.Zhang, S. Berson, S. Herzog, S. Jamin, Resource Reservation Setup Protocol, official charter maintained by the IETF, 1997-Resource ReSerVation ProtocolVersion 1 Functional Specification. RfC: 2205.

12 C. Mavromoustakis, H. Karatza, Split agent-based routing in interconnected networks (SART). International Journal of Communication Systems (IJCS), Wiley, 2004, vol. 17, pp.303-320. 